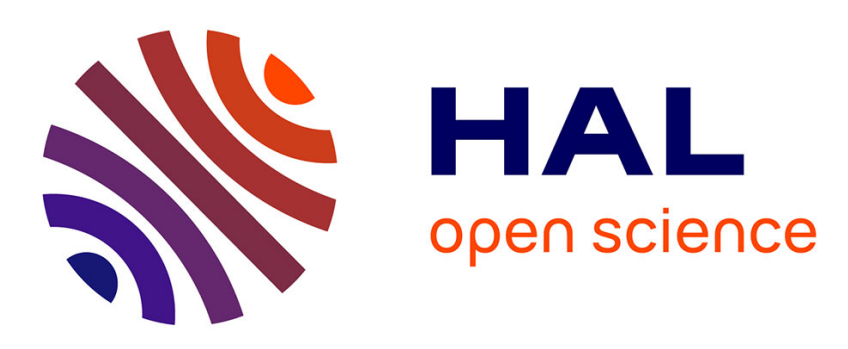

\title{
Link Availability Aware Routing Metric For Wireless Mesh Networks
}

Chiraz Houaidia, Adrien van den Bossche, Hanen Idoudi, Thierry Val, Leila Azzouz Saidane

\section{- To cite this version:}

Chiraz Houaidia, Adrien van den Bossche, Hanen Idoudi, Thierry Val, Leila Azzouz Saidane. Link Availability Aware Routing Metric For Wireless Mesh Networks. ACS/IEEE International Conference on Computer Systems and Applications - AICCSA 2013, May 2013, Fes, Morocco. pp. 1-4. hal01148081

\section{HAL Id: hal-01148081 \\ https://hal.science/hal-01148081}

Submitted on 4 May 2015

HAL is a multi-disciplinary open access archive for the deposit and dissemination of scientific research documents, whether they are published or not. The documents may come from teaching and research institutions in France or abroad, or from public or private research centers.
L'archive ouverte pluridisciplinaire HAL, est destinée au dépôt et à la diffusion de documents scientifiques de niveau recherche, publiés ou non, émanant des établissements d'enseignement et de recherche français ou étrangers, des laboratoires publics ou privés. 


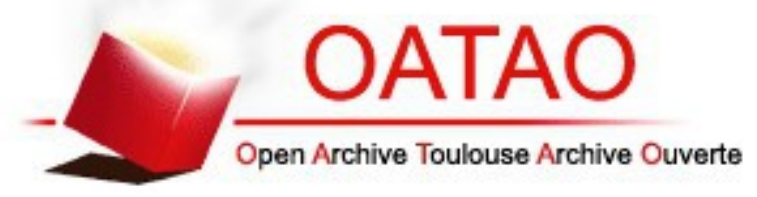

\section{Open Archive TOULOUSE Archive Ouverte (OATAO)}

OATAO is an open access repository that collects the work of Toulouse researchers and makes it freely available over the web where possible.

This is an author-deposited version published in : http://oatao.univ-toulouse.fr/ Eprints ID : 12456

To link to this article : DOI :10.1109/AICCSA.2013.6616428

URL : http://dx.doi.org/10.1109/AICCSA.2013.6616428

To cite this version : Houaidia, Chiraz and Van den Bossche, Adrien and Idoudi, Hanen and Val, Thierry and Azzouz Saidane, Leila Link Availability Aware Routing Metric For Wireless Mesh Networks. (2013) In: ACS/IEEE International Conference on Computer Systems and Applications - AICCSA 2013, 27 May 2013 - 30 May 2013 (Fes, Morocco).

Any correspondance concerning this service should be sent to the repository administrator: staff-oatao@,listes-diff.inp-toulouse.fr 


\title{
Link Availability Aware Routing Metric For Wireless Mesh Networks
}

\author{
Chiraz Houaidia*§1, Adrien Van Den Bossche ${ }^{\S 2}$, Hanen Idoudi*3, Thierry Val ${ }^{\S 4}$, Leila Azouz Saidane ${ }^{* 5}$ \\ ${ }^{*}$ National School of Computer Sciences(ENSI), \\ University of Manouba, Campus Universitaire de la Manouba, Tunisia \\ ${ }^{1}$ chiraz.houaidia@cristal.rnu.tn, $\left\{{ }^{3}\right.$ hanen.idoudi, ${ }^{5}$ leila.saidane $\} @$ ensi.rnu.tn \\ ${ }^{\S}$ CNRS-IRIT-IRT, University of Toulouse, UT2 \\ $\left\{{ }^{1}\right.$ chiraz.houaidia, ${ }^{4}$ val, ${ }^{2}$ bossche $\} @$ irit.fr
}

\begin{abstract}
This paper investigates the design of effective routing metrics in the purpose of network resources optimization and the satisfaction of users QoS requirements. Using several real experiments, we point out the shortcoming of the Expected Transmission Count (ETX) metric for eventual optimizations towards a more efficient routing. Experiments were carried out into an heterogeneous IEEE 802.11n based network running with OLSR routing protocol and have shown that ETX presents several shortcoming resulting in inaccurate estimation of the link quality and then of the routing decision. This paper presents improvements of the ETX metric based on link availibility for accurately finding high-throughput paths in multihop wireless mesh networks.
\end{abstract}

Index Terms-Wireless Mesh Network, Routing Metrics, OLSR, Testbed, IEEE 802.11n.

\section{INTRODUCTION}

Wireless Mesh Networks (WMNs) are a flexible, quickly deployable wireless networking solution that benefits from the lack of a rigid infrastructure. They are used to provide rural areas, where broadband infrastructure is not available, with a reliable Internet access based on multihop connections. Compared to adhoc networks, the main difference consists of the use of typically stationary wireless routers (backbone) to interconnect isolated LANs. These backbone nodes usually do not have strict constraints on power consumption, so, mobility and power savings are no longer the main problems in WMNs. For that reason, wireless mesh routing protocols are more and more optimized to consider link-quality metrics such as transmission capacity or error probability instead of simplistic hop-count metric used generally with adhoc networks. This new paradigm is called quality-aware routing

The design of effective routing metrics, however, must be done while optimizing network resources and satisfying users QoS requirements. It depends also on the specific characteristics of the target network. For example, due to the shared nature of the wireless medium, a wireless link in a mesh network does not have dedicated bandwidth since neighboring nodes' transmissions may also contend for the same bandwidth. Therefore, routing performance issues in a WMN are increasingly challenging and routing metrics, particularly,

This work is carried out as a part of the project tetaneutral.net must be able to capture the interference between competing flows. Packet losses, throughput degradation, congestioned links, etc., are among several problems identified in WMNs and issued generally from lower layers.

Several link-quality routing metrics [9] have been proposed and evaluated by simulations, but only a few have been implemented and evaluated in real networks [1][2]. Among implemented mesh routing metrics, we are interested to ETX [3].

The focus of this paper is to investigate the requirements for designing routing metrics in mesh networks to support high network performance, such as high throughput and low packet delay. The purpose of this paper is to setup and configure a real IEEE 802.11n [8] based WMN testbed [12] and to evaluate, in a comparative way, the OLSR performances under different metrics.

The remainder of this paper is organized as follows: We describe some routing metrics in section II mainly the ETX metric. Section III presents a measurement based evaluation of ETX metric in order to point out its shortcoming and ways towards its optimization. We finally present some improvements of ETX metric in section IV.We conclude by Section V.

\section{RELATED WORK}

The design of effective routing metrics depends on the characteristics of the target network. For example, the severe energy constraints of sensor networks require the design of energy efficient routing metrics. Such metrics respect the emergency character of some applications and the resources availability.

In ad hoc networks, where mobility and topology changes are the main problems, the most convenient metric is hop count. This metric, coupled with a routing strategy, allows a fast recovery of instable routes due to link breakage. However, this shortest-path routing approach implicitly assumes that links either work perfectly or do not work at all.

For wireless mesh backbone, where routers are relatively stationnary and the wireless medium is shared, mesh routing protocols are optimized to consider link quality metrics in order to ensure high throughput and low packet delay.

New metrics, such as ETX, ETT, WCETT, MIC, etc. [9], are proposed towards a quality-aware routing, in order to reflect 
more the link variations such as transmission capacity, loss probability, interferences, etc.

The research community has a particular interest in ETX metric [3]. Indeed, this metric has been the subject of several performance studies and several implementations and integrations in real platforms. In this work, we mainly focus on this research effort and rely on the study of the limitations of this metric and propose improvements.

The Expected Transmission Count (ETX) metric is a proposal to find the route with the highest probability of packet delivery. It represents the number of times a node expects to transmit and retransmit a packet for a successfull delivery. The ETX metric considers the asymetric property of wireless links and is computed as follows :

$$
E T X=1 /\left(d_{f} \times d_{r}\right)
$$

Where $d_{f}$ and $d_{r}$ are respectively the forward and the reverse delivery ratios of the link.

To estimate $d_{f}$ and $d_{r}$, nodes broadcast small-size probes at an average period $\tau$ during a time window $\omega$ so that each node knows how much probes it should receive during this period which is $\tau / \omega$. The probe contains the number of received probes for each neighbor during the last $\omega$. So, each receiving node becomes aware of the forward delivery ratio for each link. This information is then diffused to make all neighbors aware of the ETX of the link. The best link quality is the link with the smallest ETX i.e with the smallest loss probability. The ETX of a route is the sum of the link metrics.

This metric was well studied by real experiments under different testbeds [1][2]. One of the most popular implementations is that with OLSR. One of the most-known implementations of OLSR [7] is OLSRd program (OLSR daemon) [13] which implements basically hop count and ETX as metric.

\section{TestBed And ExPERImEntal Results}

As part of this work, we conducted a series of tests in order to evaluate OLSR-ETX performances in a real IEEE $802.11 \mathrm{n}$ based wireless mesh network and accurately adress the limitations of such metric.

We run experiments in an indoor testbed formed by seven nodes: four shuttles, two personal computers and one nanostation router. Device configurations are shown in Table I. All nodes are $2 \times 2$ MIMO devices equipped with the ath9k driver and are using OpenWRT operating system. The included driver provides enough features for our implementation as well as for future improvements. All nodes are configured to use the channel 9 in the IEEE 802.11n mode. The OLSR routing protocol is implemented natively in the OpenWRT operating system as an OLSR deamon (olsrd). This implementation is improved to offer both the use of the Hop Count and the ETX metrics. Our testbed called BlueMeLab [12] is deployed at the University Institute of Technology (IUT) Blagnac-Toulouse. The nodes are spread over two floors of the building (cf. Figure $1)$. Rooms are separated by thick bricked walls. It is worthy mentioning that there are other wireless networks in the area.
The choice, then, of the channel was made essentially in order to avoid interferences with existant networks and garantee a certain accuracy of our results.

\section{TABLE I: CONFIGURATION OF DEVICES USED ON THE TESTBED}

\begin{tabular}{|c|c|c|c|}
\hline Device & Operating System & Processor & RAM \\
\hline Computer & $\begin{array}{c}\text { OpenWRT } \\
\text { (GNU/Linux) }\end{array}$ & $\begin{array}{c}\text { Intel Pentium 4 } \\
\text { CPU 3.2GHz }\end{array}$ & $2 \mathrm{Go}$ \\
\hline Shuttle & $\begin{array}{c}\text { OpenWRT } \\
\text { (GNU/Linux) }\end{array}$ & $\begin{array}{c}\text { Intel Pentium 4 } \\
\text { CPU 3.00GHz }\end{array}$ & 904Mo \\
\hline $\begin{array}{c}\text { NanoStation } \\
\text { Router }\end{array}$ & $\begin{array}{c}\text { OpenWRT } \\
\text { (GNU/Linux) }\end{array}$ & $\begin{array}{c}\text { Atheros MIPS } \\
180 \mathrm{MHz}\end{array}$ & $\begin{array}{c}\text { 16MB SDRAM } \\
\text { 4MB Flash }\end{array}$ \\
\hline
\end{tabular}

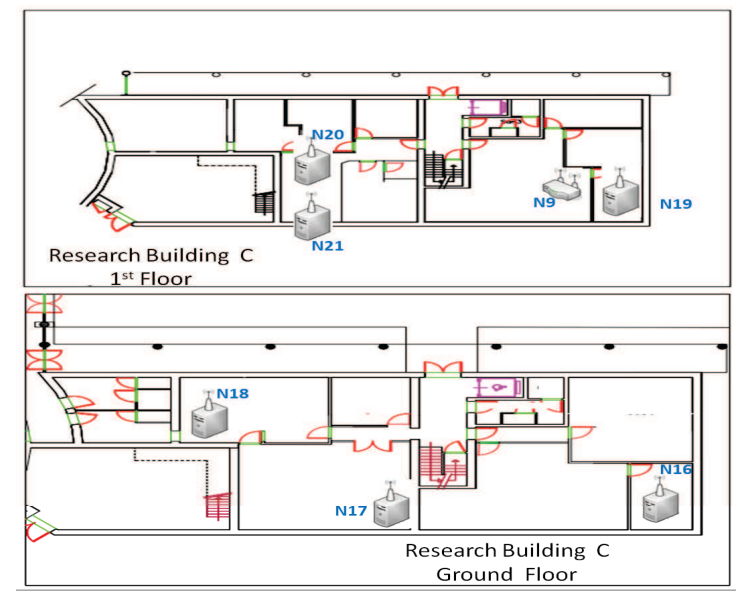

Fig. 1: The Testbed

As mentioned above, olsrd implements natively the hop count and the ETX metrics. To measure ETX, olsrd, instead of creating new probe messages, uses HELLO and TC messages of OLSR which are sent periodically each $2 \mathrm{~s}$ and 5 s respectively. This method aims to avoid extra overhead. A multitude of monitoring tools were offered by the ath9k driver and used in our experiments. The iperf tool was used to generate TCP and UDP traffic at different rates between pairs of nodes and measure bandwidth, jitter, packet loss, etc. tcpdump and Wireshark tools were used to dump the traffic in the network and give a description of the contents of packets. iw configuration utility was used to get device capabilities and set parameters and statistics. All the performance evaluation in this paper are the result of measurements taken on the wireless testbed presented hereby.

With ETX metric, the link quality estimation is based on small probe size (some bytes) which doesn't properly reflect the data loss probability. In fact, such measurement underestimates data loss ratios and over-estimates ACK loss ratios. In real scenarios, the packet loss rate and delay are largely higher while increasing the traffic load or the packet size. Moreover, ETX link probes are susceptible to MAC unfairness and route ETX measurements may change under 
load. These observations are detailed by experimentations in a previous work [11].

On the other hand, ETX assumes all links run at one bit-rate and probes are sent in broadcast at the network basic physical rate. So, this metric assumes a robust physical layer which is not the case at all. When links data rates are not accounted for, a short path with lower ETX may be chosen over another longer path with higher ETX albeit the latter may be able to support a higher overall throughput and less end-to-end delay. Based on the topology below (cf. Figure 2), we demonstrate the limitations of ETX Sum using experiments of four different paths.

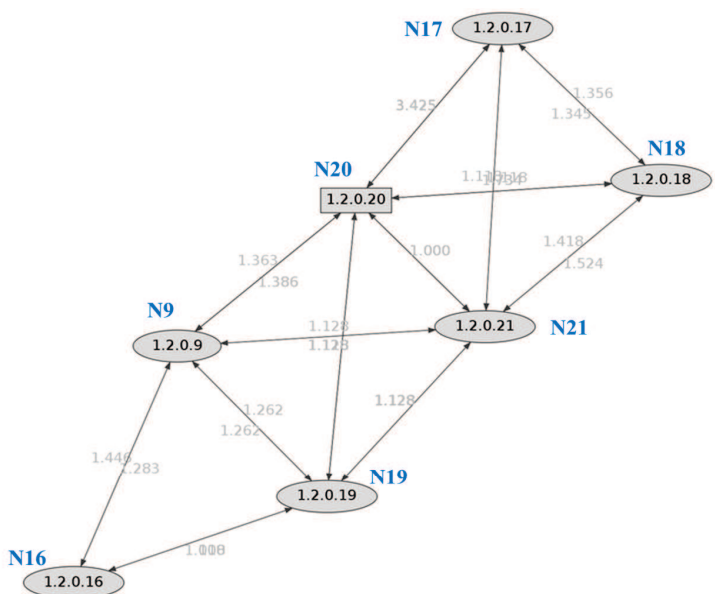

Fig. 2: The Testbed Topology

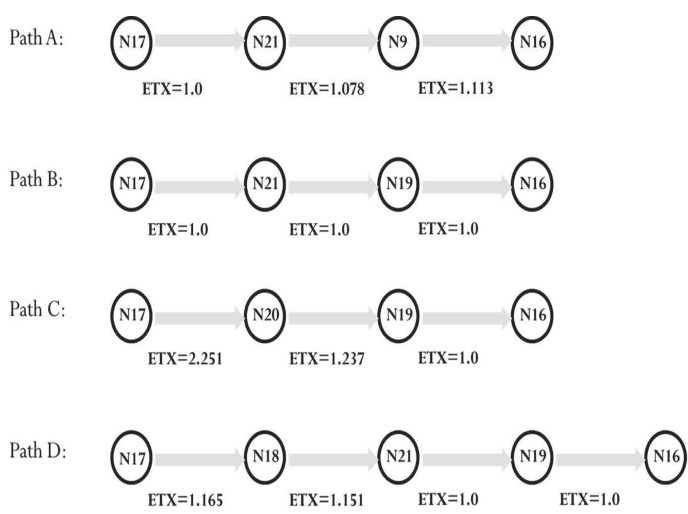

Fig. 3: Four Ad hoc Paths

TABLE II: RESULTS OF EXPERIMENTATIONS

\begin{tabular}{|c|c|c|}
\hline Path & ETX SUM & Throughput (Mbits/sec) \\
\hline PathA & 3.191 & 15.12 \\
\hline PathB & 3.000 & 6.5 \\
\hline PathC & 4.488 & 5.74 \\
\hline PathD & 4.316 & 6.2 \\
\hline
\end{tabular}

Consider the four ad hoc paths shown in Fig.3 where the send rate of each source node is the maximal one-hop data rate of IEEE 802.11n. TCP traffic is used to get the throughput achieved by each path. First, consider Path A and Path B. Both are 3-hop paths but the ETX Sum of Path A is larger than that of Path B. According to OLSR-ETX, Path B will be selected as the better route to reach node N16 from node N17 while, in reality, the throughput of Path A is approximately $230 \%$ higher than that of Path B.

Second, consider Path C and Path D. Path C is 3-hop route but because of the bad quality of the link (N17, N20), its ETX Sum is higher than that of Path D which is 4-hop route. According to OLSR-ETX, Path D is selected whenever it is longer.

These simple examples show that an ETX Sum metric cannot accurately differentiate paths with different achievable data rates. The main reason is that, ETX is an interference insensitive metric. In fact, when successive hops have different loss rates, the transmission interference of the two nodes is different. The changed transmission interference considerably affects the achievable data rates. ETX Sum is unable to capture this phenomenon.

Further more, a total Sum of ETXs is too simplistic and not always efficient. A route with an ETX equal to $3(\mathrm{ETX}=3.0)$ doesn't mean that this route is made of three hops nor that it is longer than a route with an ETX equal to $2(\mathrm{ETX}=2.0)$.

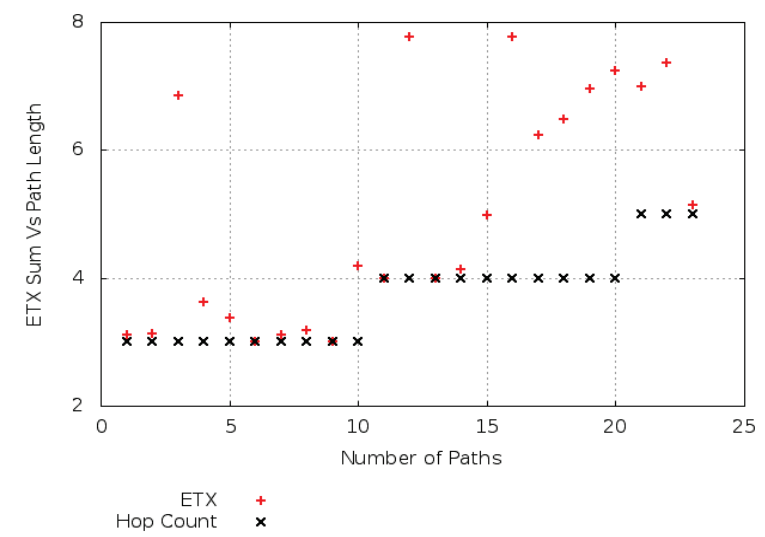

Fig. 4: ETX SUM Vs Path length

Fig. 4 shows the ETX Sum and hop length of each path from 42 total paths according to our topology. We find that, for nhop paths, ETX Sum is between $\mathrm{n}$ and $2 \mathrm{n}$. Particularly for paths longer than four hops, their ETX Sums are quite larger than their hop lengths.

\section{ETX IMPROVMENTS}

To overcome the limitations of ETX, a first step would be to take into account the aspect of "multi-rate" in the choice of the link. Indeed, traffic should be routed into the best highspeed links in order to achieve the best possible end-to-end throughputs and delays. 
Another aspect one should consider is the link availability and/or occupancy to propose a load-sensitive and loadbalancing routing. In fact, based on the occupancy estimation of a link, the routing protocol would avoid congestioned routes and select links which are "freer" and the most available to transmit data traffic in order to garantee reliability. Such information reflects the possible congestion or interference phenomenon that may affect the data packet delivery.

Using this information of link occupancy, only links with reduced occupancy are selected to form a route between a source node and a destination node so that routing decision is based on link's availability to support more traffic flows. Bottelneck or lossy links with high occupancy rates are supposed to bad links.

Since the link occupancy information is inable to measure accurately the capacity of a link to support a specific amount of data, we should account for the residual link capacity to adapt the transmission relatively to the link capacity.

\section{CONCLUSion And Future WORK}

This paper presents the shortcomings of ETX and the challenging aspects in the design of an effective routing metric for WMNs. By taking into account the PHY/MAC diversity of nodes, we focused on the shortcomings of these metrics to adapt a heterogeneous environment.

This paper provides a measurement-based performance evaluation of the OLSR protocol using different metrics such as hop-count and ETX. Results have shown the inability of ETX to measure accurately the link quality and then the routing decision. Results obtained are related to the considered topology, further study of other topologies is needed to validate this conclusion. Although, our results remain coherent with other works [2][6].

We presented, then, improvements and optimizations of this metric towards a link availibility aware routing. Our improvements are load-sensitive and aim to fairly distribute the traffic load between nodes in the network while taking into account their occupancy and availability.

Future work will consist of the proposal of a link availibility aware routing metric and an adaptive routing protocol taking into account this metric. Further contributions will be validated by simulation, prototyping and then deployed on the real mesh network deployed tetaneutral.net[14] in Toulouse, France which offers a more realistic environment, traffic and wireless contraints. It allows us also to check the scalability of our proposal when deployed in a large scale network.

\section{ACKNOWLEDGMENT}

This work is carried out as a part of the project tetaneutral.net. Further contributions will be tested in the real network. Authors would like to thank the project's manager support, Laurent Guerby.

\section{REFERENCES}

[1] M. E. M. Campista, D. G. Passos, P. M. Esposito, I. M. Moraes, C. V. N de Albuquerque, D. C. M. Saade, M. G. Rubinstein, L. H. M. K. Costa and O. C. M. B. Duarte. "Routing metrics and protocols for wireless mesh networks" IEEE Network, vol. 22, no. 1, pp. 6-12, Jan.-Feb. 2008.
[2] P. M. Esposito, M. E. M. Campista. , I. M. Moraes, L. H. M. K. Costa, O. C. M. B. Duarte, M. G. Rubinstein. "Implementing the Expected Transmission Time Metric for OLSR Wireless Mesh Networks". IFIP Wireless Days, Dubai, Nov. 2008, pp. 1-5.

[3] D. S. J. D. Couto, D. Aguayo, J. Bicket, and R. Morris. "A highthroughput path metric for multi-hop wireless routing". ACM MobiCom, California, Sept. 2003, pp. 134-146.

[4] R. Draves, J. Padhye, and B. Zill. "Routing in multi-radio, multi-hop wireless mesh networks". ACM MobiCom, Philadelphia, Sept. 2004, pp. 114-128.

[5] D. Aguayo, J. Bicket, and R. Morris. "SrcRR: A high throughput routing protocol for 802.11 mesh networks (DRAFT)". MIT, Tech. Rep., 2005.

[6] H. Sinky and B. Hamdaoui. "Implementation and performance measurement and analysis of OLSR protocol". International Wireless Communications and Mobile Computing Conference (IWCMC), New York, Jul. 2010. pp. 286-290.

[7] T. Clausen and P. Jacquet. "Optimized Link State Routing Protocol (OLSR)”. Internet Request For Comments RFC 3626, Internet Engineering Task Force, Oct. 2003.

[8] C. Wang and H. YuWei. "IEEE 802.11n MAC Enhancement and Performance Evaluation". Mobile Networks and Applications, vol. 14, no. 6,Dec. 2009, pp. 760-771.

[9] S. Ghannay, S.M. Gammar, F. Filali, F. Kamoun. "Multi-radio multichannel routing metrics in IEEE 802.11s-based wireless mesh networks - And the winner is ". Communications and Networking (ComNet), Hammamet, Nov. 2009, pp.1-8.

[10] C. Houaidia, H. Idoudi, A. Van Den Bossche, T. Val, L. Saidane. "Impact of IEEE 802.11 PHY/MAC Strategies on Routing Performance in Wireless Mesh Networks". FINA International Symposium in conjunction with AINA, Barcelona, Mar. 2013.

[11] C. Houaidia, A. Van Den Bossche, H. Idoudi, T. Val, L. Saidane. "Experimental Performance Analysis of Routing Metrics in Wireless Mesh Networks". Multihop Wireless Network Testbeds and Experiments workshop in conjunction with IWCMC, Cagliari, Jul. 2013.

[12] http://lab.iut-blagnac.fr/bluemelab. Last access: March, 2013.

[13] http://www.olsr.org. Last access: March, 2013.

[14] http://tetaneutral.net. Last access: March, 2013. 